\title{
Effects of enriched endogenous omega-3 fatty acids on age-related hearing loss in mice
}

\author{
Yohei Honkura $^{1 \dagger}$, Jun Suzuki ${ }^{1 *}$ (D), Nobuyuki Sakayori ${ }^{2}$, Hitoshi Inada ${ }^{3}$, Tetsuaki Kawase ${ }^{1,4}$, Yukio Katori $^{1}$ \\ and Noriko Osumi ${ }^{3}$
}

\begin{abstract}
Objective: Dietary intervention is a practical prevention strategy for age-related hearing loss (AHL). Omega-3 ( $n-3$ ) polyunsaturated fatty acids (PUFAs) may be effective in prevention of AHL due to their anti-inflammatory and tissueprotective functions. Age-related changes in the hearing function of wild-type and Fat- 1 transgenic mice derived from the C57BL/6N strain, which can convert omega-6 PUFAs to n-3 PUFAs and consequently produce enriched endogenous n-3 PUFAs, were investigated to test the efficacy of n-3 PUFAs for AHL prevention.

Results: At 2 months, the baseline auditory brainstem response (ABR) thresholds were the same in Fat-1 and wild-type mice at 8-16 kHz but were significantly higher in Fat-1 mice at 4 and $32 \mathrm{kHz}$. In contrast, the ABR thresholds of Fat-1 mice were significantly lower at 10 months. Moreover, the ABR thresholds of Fat- 1 mice at low-middle frequencies were significantly lower at 13 months $(12 \mathrm{kHz})$. Body weights were significantly reduced in Fat-1 mice at 13 months, but not at 2, 10, and 16-17 months. In conclusion, enriched endogenous n-3 PUFAs produced due to the expression of the Fat- 1 transgene partially alleviated AHL in male C57BL/6N mice.
\end{abstract}

Keywords: Omega-3 (n-3) fatty acids, Cochlea, Age-related hearing loss, C57BL/6 mouse

\section{Introduction}

Age-related hearing loss (AHL) is the most common cause of sensorineural hearing loss in adults and is one of the most prevalent age-related physical conditions $[1,2]$. There are currently no established preventions or treatments for AHL, even though it is a high priority issue. Nutritional improvement is one potential intervention, and omega-3 (n-3) polyunsaturated fatty acids (PUFAs), such as docosahexaenoic acid (DHA), are promising candidates for AHL prevention due to their potential prevention of cognitive decline [3]. In addition, anti-inflammatory and pro-resolving metabolites of $n-3$ PUFAs are known to have protective effects in neurological disorders [4].
*Correspondence: j_suzuki1212@orl.med.tohoku.ac.jp

${ }^{\dagger}$ Yohei Honkura and Jun Suzuki contributed equally to the work

${ }^{1}$ Department of Otolaryngology-Head and Neck Surgery, Tohoku University School of Medicine, 1-1 Seiryo-machi, Aoba-ku, Sendai, Miyagi 980-8574, Japan

Full list of author information is available at the end of the article
Several studies have reported positive effects of $n-3$ PUFAs on hearing function in humans, [5-7] while perinatal diets supplemented with high levels of DHA or n-3 PUFAs have negative effects on the auditory systems of rat pups [8, 9] and adult rat offspring [10]. Therefore, the effects of n-3 PUFAs on hearing function remain unclear, particularly due to the unavoidable genetic and environmental non-uniformity of human subjects.

In this study, we investigated the preventive effects of enriched endogenous n-3 PUFAs on the progression of AHL in Fat-1 transgenic mice (Fat-1 mice). The Fat-1 mice express the nematode-derived Fat-1 gene encoding an enzyme to convert omega-6 (n-6) to n-3 PUFAs [11]. Thus, investigation of Fat-1 mice provides more reliable and more definitive results than the studies using conventional dietary supplementation of PUFAs.

\section{Main text \\ Methods \\ Animals and genotyping}

Wild-type (WT) C57BL/6N mice were purchased from CLEA Japan. Heterozygous Fat-1 mice [11] were mated 
with WT mice, and their male offspring were used. Mice were maintained on a normal diet (CE-2, CLEA Japan, Tokyo, Japan) with water ad libitum and housed under a standard $12 \mathrm{~h}$ light $/ 12 \mathrm{~h}$ dark schedule.

Genotyping was performed as previously described [12]. The following primers were used to amplify the Fat-1 transgene: forward 5'-CACCAACCACATCGA CAAAG-3' and reverse 5'-CGACGTGCTGCAGATAGG TA-3'. Polymerase chain reaction amplification was performed for 30 cycles under the following parameters: denaturation at $95^{\circ} \mathrm{C}$ for $30 \mathrm{~s}$, annealing at $55^{\circ} \mathrm{C}$ for $30 \mathrm{~s}$, and extension at $72{ }^{\circ} \mathrm{C}$ for $2 \mathrm{~min}$.

\section{Cochlear function testing}

Auditory brainstem responses (ABR) were recorded as previously described [13]. The animals were anesthetized with intraperitoneal injection of ketamine $(100 \mathrm{mg} / \mathrm{kg})$ and xylazine $(20 \mathrm{mg} / \mathrm{kg})$. Needle electrodes were placed subcutaneously at the vertex, the base of the pinna, and the back. ABR recordings were obtained using a TDT System 3 hardware and BioSigRP software (Tucker-Davis Technologies, Alachua, FL). ABRs were evoked with tone bursts of pure tones at frequencies of $4,8,12,16$, and $32 \mathrm{kHz}$, which were generated using SigGenRP software and a digital-to-analog converter (RP2.1). ABRs were relayed to a programmable attenuator (PA5), an amplifier (SA1), and a closed-field loudspeaker (CF1). The electrode outputs were delivered to an alternating current preamplifier (P55, Astro-Med, West Warwick, RI) and amplified $(\times 100)$. Evoked responses were filtered with a band pass of $10-3000 \mathrm{~Hz}$ and were averaged with 1000 sweeps. Responses were collected for stimulus levels in 5-dB steps from sound pressure levels (SPLs) of $100 \mathrm{~dB}$ to $10 \mathrm{~dB}$. ABR threshold was defined as the lowest sound level at which reproducible waveforms could be observed. If no response was obtained at $100 \mathrm{~dB}$ SPL, the ABR threshold was defined as $105 \mathrm{~dB}$ SPL.

\section{Tissue preparation}

The mice were anesthetized with an intraperitoneal injection of ketamine $(100 \mathrm{mg} / \mathrm{kg})$ and xylazine $(20 \mathrm{mg} /$ $\mathrm{kg}$ ). The anesthetized mice were then transcardially perfused with 4\% paraformaldehyde (P6148, Sigma-Aldrich, St. Louis, MO) in phosphate-buffered saline (PBS). The cochleae were removed and post-fixed in $4 \%$ paraformaldehyde overnight at $4{ }^{\circ} \mathrm{C}$. The fixed cochleae were decalcified in 10\% ethylenediaminetetra-acetic acid disodium salt dehydrate (345-01865, Dojindo, Mashiki, Japan) for 2 days at $4{ }^{\circ} \mathrm{C}$.

\section{Cochlear whole-mount}

Microdissected cochlear pieces were blocked in 5\% normal horse serum in PBS and 0.3\% Triton X-100 for
$10 \mathrm{~min}$ at room temperature and stained with a rhodamine-phalloidin probe (1:250, Cytoskeleton, Denver, $\mathrm{CO})$ at room temperature for $30 \mathrm{~min}$. Cochlear pieces were slide-mounted using Vectashield (Vector Labs, Burlingame, CA). Cochlear pieces were imaged using a fluorescence microscope (E800, Nikon, Tokyo, Japan). Stained cochlear whole-mounts were imaged using confocal microscopy (TCS SP5, Leica, Wetzlar, Germany). Quantitative results were obtained by evaluating 90 outer hair cells $(\mathrm{OHCs})$ across three rows in a given microscopic field.

\section{Histological analysis of spiral ganglion (SG) neurons and stria vascularis (SV)}

Decalcified tissues were embedded in paraffin, and coronal Sects. $(3 \mu \mathrm{m})$ were cut and mounted. The sections were stained with hematoxylin and eosin and visualized using a light microscope (BZ-9000, Keyence, Osaka, Japan). Three cochlear regions were used for evaluation of cochlear histology. Three sections per animal were used to calculate mean numbers. Area measurements and cell counts were performed using BZ-H1C (Keyence). The SG area was calculated for each section, and the SG neurons were counted. Three measurements of SV thickness were obtained from each image and were averaged.

\section{Statistical analysis}

Statistical analyses were conducted using StatMate IV (ATMS Company, Tokyo, Japan) or JMP ${ }^{\circledR} 12$ (SAS Institute Inc., Cary, NC). All data are presented as means \pm standard errors of the mean. The two-sample $t$-tests and two-way analyses of variance (ANOVA) followed by Bonferroni post hoc tests were used. $P$ values $<0.05$ after the Bonferroni correction were considered statistically significant.

\section{Results}

\section{Time course of ABR thresholds and body weights}

The C57BL/6N mice exhibit early onset and progression of AHL mainly due to a single nucleotide variant in the $C d h 23$ gene $\left(C d h 23^{753 \mathrm{~A}}\right)[14,15]$. Fat-1 mice have a $C d h 23^{753 \mathrm{~A} / 753 \mathrm{~A}}$ genotype for $C d h 23$ (Additional file 1: Fig S1). To determine the effects of enriched endogenous n-3 PUFAs on AHL progression, ABR thresholds were first measured at 2,13 , and 16-17 months of age (Fig. 1a). Although there were no differences in the ABR thresholds in response to $8-, 12-$, or $16-\mathrm{kHz}$ stimuli, the ABR thresholds were significantly higher in response to $4-\mathrm{kHz}$ and $32-\mathrm{kHz}$ stimuli in Fat-1 mice at 2 months (Fig. 1b). Unlike at 2 months, the ABR thresholds at 13 months were significantly lower at $12 \mathrm{kHz}$ in Fat-1 
a

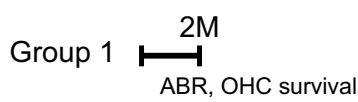

Group $2 \underbrace{13 M}_{A B R, S G N \text { number, SV thickness }}$

16-17M

Group 3

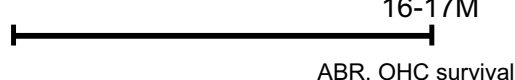

C

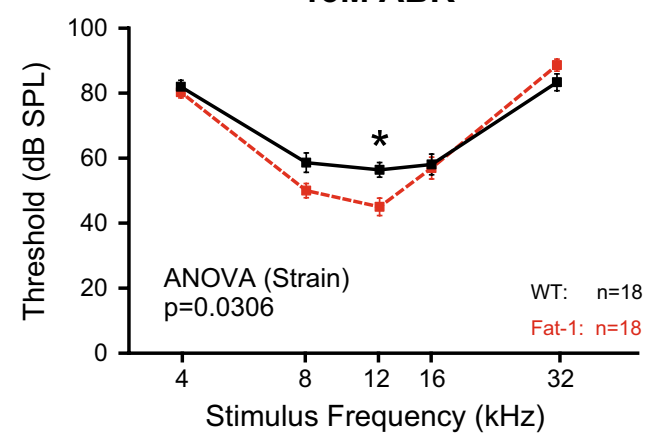

e

Body weight (g)

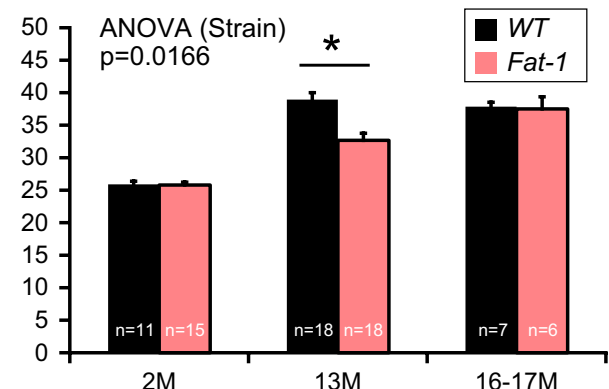

b

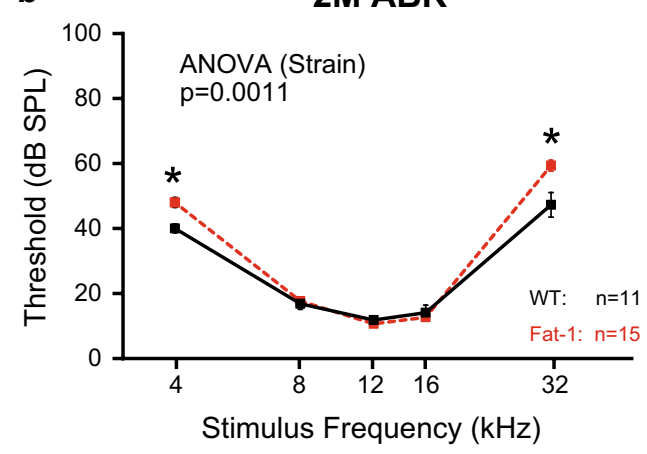

d

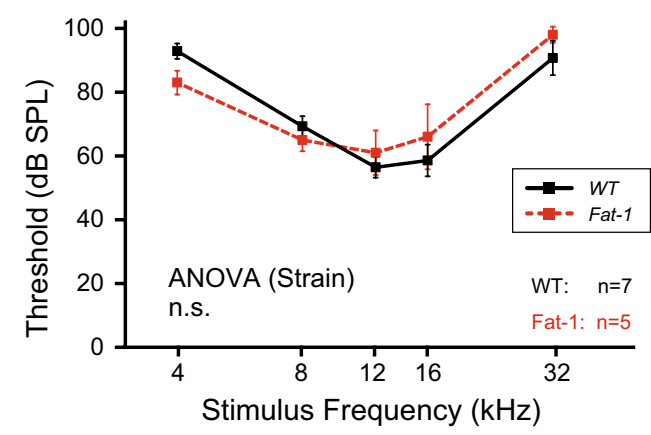

Fig. 1 Chronological changes in auditory brainstem response (ABR) thresholds and body weights. a Schematic timeline of the experimental protocol. b-d Mean ABR thresholds ( \pm standard errors of the mean) for each time point (b 2 months, c 13 months, and d 16-17 months). Animal numbers were as follows: Wild-type (WT), $n=11$; Fat $-1, n=15$ in $\mathbf{b}$ WT, $n=18 ;$ Fat- $1, n=18$ in $\mathbf{c}$; and WT, $n=7 ;$ Fat-1, $n=5$ in $\mathbf{d}$. In the early stage (2 months), ABR thresholds were significantly elevated in Fat-1 mice compared with WT mice in response to stimuli at 4 and $32 \mathrm{kHz}$ (b). In contrast, ABR thresholds of Fat-1 mice were significantly decreased at low-middle frequencies at 13 months (c). Time course of changes in body weight at 2 months (wild-type [WT], $\mathrm{n}=11$; Fat-1, $\mathrm{n}=15), 13$ months (WT, $\mathrm{n}=18$; Fat-1, $\mathrm{n}=18$ ), and 16-17 months (WT, $\mathrm{n}=7 ;$ Fat-1, $\mathrm{n}=6)$ of age. e Body weights of WT and Fat-1 mice were not different in the early stage, but those of Fat-1 mice were significantly decreased at 13 months. No significant difference was observed between the two groups in the later stage (at 16-17 months). $P$ values $<0.05$ were considered statistically significant $(*)$. OHC outer hair cell, SGN spiral ganglion neuron, SPL sound pressure level, SV stria vascularis, ANOVA analysis of variance, ns no significant difference

mice (Fig. 1c). No significant differences were observed at 16-17 months (Fig. 1d).

WT and Fat-1 mice in the same colony were weighed at 2,13 , and 16-17 months to evaluate the effect of enriched endogenous n-3 PUFAs on body weight, Although the body weights of WT mice increased and reached a plateau at 13 months, the body weights of Fat-1 mice increased more slowly and reached the same level as that reached by WT mice at $16-17$ months (Fig. 1e). The body weights of Fat-1 mice were significantly lower than those of WT mice at 13 months (Fig. 1e). Therefore, enriched endogenous n-3 PUFAs slowed age-related weight gain. 
We additionally evaluated the ABR thresholds at 10 months using a different colony. Although ABR thresholds for both WT and Fat-1 mice were increased compared to those of other groups (13 and 16-17 months) due to unknown reasons, we found that the ABR thresholds were significantly different between the two strains at 10 months (Fig. 2a, Additional file 1: Tables S1). These results suggest that enriched endogenous n-3 PUFAs delayed the early progression of AHL.

\section{OHC survival rates at 2 and 16-17 months}

Representative cochlear whole-mount images from WT and Fat-1 mice in the $32-\mathrm{kHz}$ region at 2 and 16-17 months are shown in Fig. 3a-d. Almost all OHCs were intact in both WT and Fat-1 mice at 2 months (Fig. 3a, b). In contrast, OHCs were severely damaged in both WT and Fat-1 mice at 16-17 months (Fig. 3c, d). OHC survival rates were not significantly different between these two groups at 2 months and 16-17 months (Fig. 3e, f). In summary, OHC survival did not significantly differ between the two groups at young (2 months) and old (16-17 months) stages.

\section{Histological changes in cochleae at 2 and 13 months}

Cochlear coronal sections were histologically investigated at 2 and 13 months to assess the effects of enriched endogenous $n-3$ PUFAs on cochlear degeneration. No apparent cochlear degradations were observed in the SG, SV, or spiral ligament of WT or Fat-1 mice at 2 months (Fig. 3g, h). Although several indicators of cochlear degeneration were observed, no apparent differences were found between WT or Fat-1 mice at 13 months (Fig. 3i, j). The number of SG neurons and the thickness of the SV showed no significant differences between the two groups at 13 months (Fig. 3k, l). Therefore, no apparent differences in age-related cochlear degeneration were found between WT and Fat-1 mice at 13 months.

\section{Discussion}

Dietary intervention may be a promising prophylaxis for AHL, as diet is one modifiable risk factors for AHL [16]. Here we used Fat-1 mice to evaluate the effects of n-3 PUFAs without the many problems associated with dietary supplementation such as oxidation of PUFAs in food pellets. We found that enriched endogenous n-3 PUFAs suppressed age-related body weight gain and partially slowed the progression of AHL in male C57BL/6N mice. Recently, long-term dietary n-3 PUFA supplementation was shown to ameliorate the progression of AHL in female C57BL/6J mice [17]. In that study, ABR thresholds in response to $4-, 8-$, and $40-\mathrm{kHz}$ stimuli were significantly lower in mice administered n-3 PUFA at 10 months [17]. This protective effect during the early aging period seems to be reproducible, as we also observed a similar protective effect in male Fat-1 mice at 10 months (Fig. 2a). Together, these observations may suggest that $\mathrm{n}-3$ PUFAs are effective for AHL prevention in C57BL/6 mice during the early aging period.

In this study, the progression of AHL was significantly slowed in Fat-1 mice only in response to low-middle frequencies. This may have been due to baseline differences in $\mathrm{ABR}$ threshold at 2 months. Considering that the ABR thresholds of Fat -1 mice at 10 months tended to be lower than those in WT mice (Fig. 2a), enriched endogenous n-3 PUFAs may have protective effects on a wider range of cochlear frequency regions. However, it is also possible
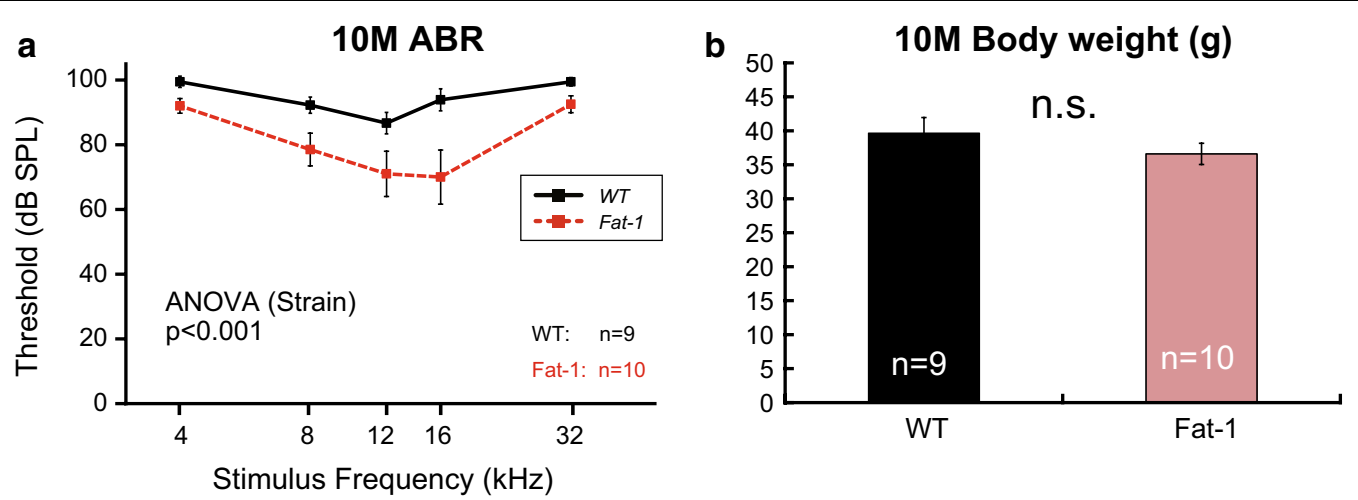

Fig. 2 Auditory brainstem response (ABR) thresholds and body weights at 10 months of age. a Mean ABR thresholds ( \pm standard errors of the mean) at 10 months. ABR thresholds for both wild-type (WT) and Fat-1 transgenic mice (Fat-1) were increased when compared to those of other groups (13 and 16-17 months) due to unknown reasons. The animal numbers were as follows: WT, $n=9$ and Fat-1, n=10. Although there were no significant differences in stimulus frequency as indicated in the Bonferroni post hoc test, the two-way analysis of variance (ANOVA) revealed that the main effect (strain) was statistically significant. b Body weights at 10 months (WT, $\mathrm{n}=9 ;$; Fat-1, $\mathrm{n}=10$ ). $P$ values $<0.05$ were considered to statistically significant $(*)$. ns no significant difference 

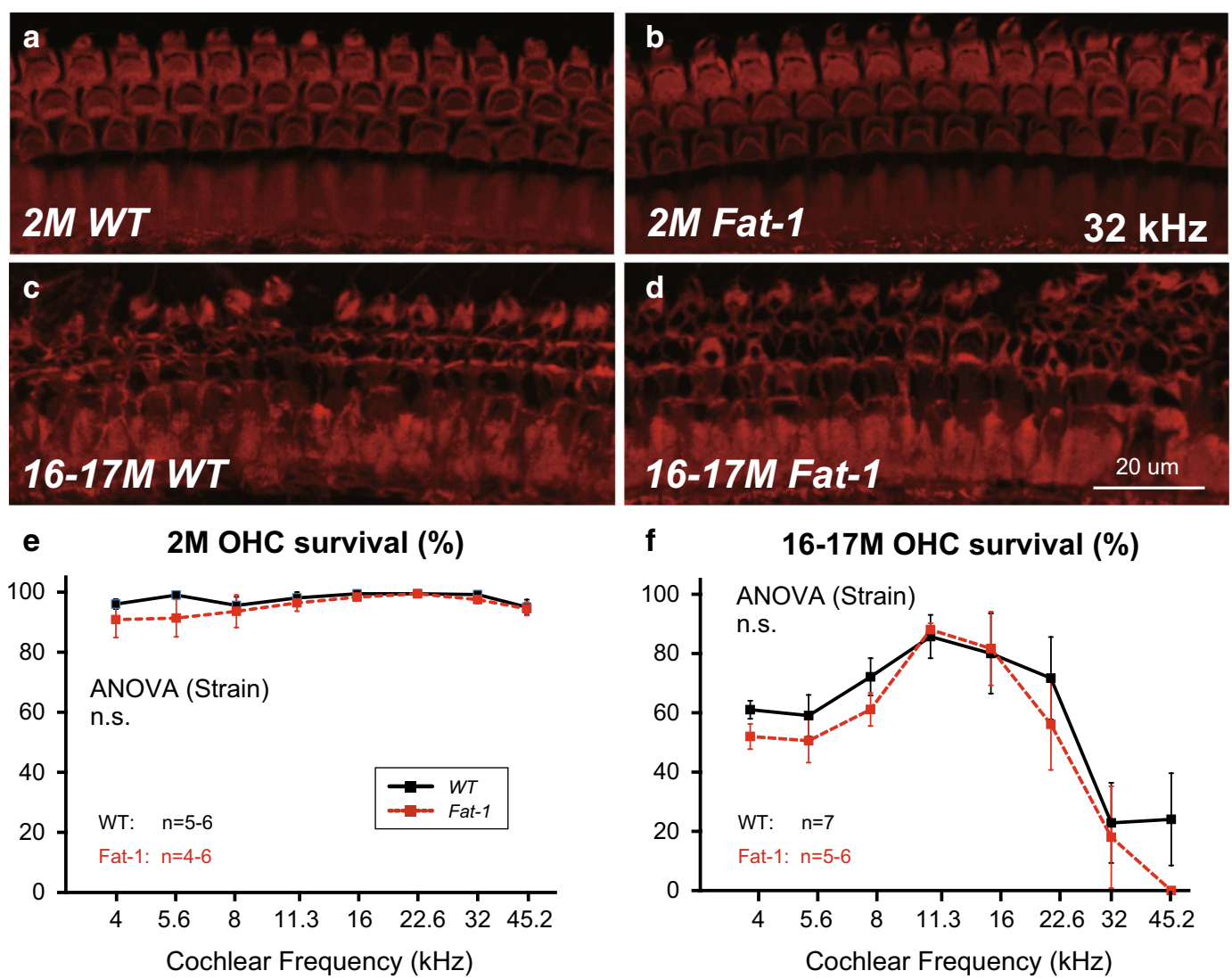

\section{f $\quad$ 16-17M OHC survival (\%)}
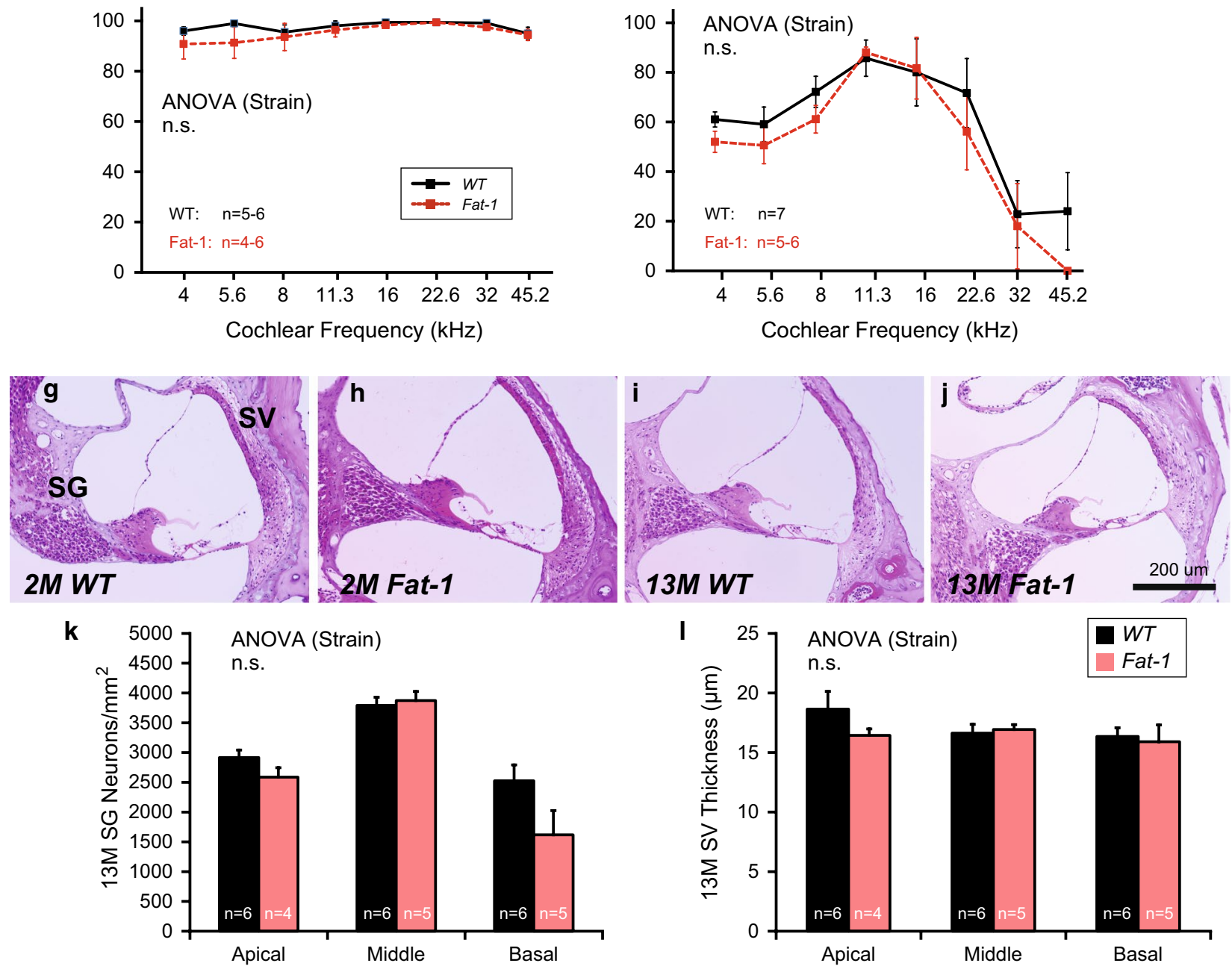

Fig. 3 Cochlear histology. a-d Representative images of organs of Corti in the 32-kHz region [a wild-type (WT) mice at 2 months, $\mathbf{b}$ Fat-1 mice at 2 months, cWT mice at 16-17 months, and $\mathbf{d}$ Fat-1 mice at 16-17 months]. At $32 \mathrm{kHz}$, almost all OHCs were intact in the early stage (a, b), but were severely damaged at 16-17 months in both WT and Fat-1 mice (c, d). e Quantitative data for OHC survival at 2 months. $\mathbf{f}$ Quantitative data of OHC survival at 16-17 months. $\mathbf{g}$-j Representative images of the cochlear middle turn (g wild-type [WT] mice at 2 months, $\mathbf{h}$ Fat- 1 mice at 2 months, $\mathbf{i}$ WT mice at 16-17 months, and $\mathbf{j}$ Fat-1 mice at 16-17 months). There were no apparent differences between these two groups at either 2 or 13 months. k Quantitative data for spiral ganglion (SG) neuron counts at 13 months. I Quantitative data for stria vascularis (SV) thickness measurements at 13 months. $P$ values $<0.05$ were considered statistically significant $\left.{ }^{*}\right)$. ns no significant difference 
that sensitivity to n-3 PUFAs varies in different cochlear regions. We have already observed such a regional difference: maternal consumption of a diet high in n- 6 and deficient in $\mathrm{n}-3$ has been reported to severely impair neocortical development in the rostral region, although the effect is mild in the caudal region [12]. n-3 PUFA receptors and transporters may have a gradient expression in the cochlea. Further investigation is required to increase our knowledge of PUFA-mediated molecular mechanisms in the cochlea, and Fat-1 mice will be useful for future studies.

In addition to the positive effects of enriched endogenous n-3 PUFAs for AHL prevention, we also noticed negative effects on hearing development (Fig. 1b). These negative effects of n-3 PUFAs are consistent with previous studies using rats: maternal dietary supplementation of DHA had negative effects on auditory brainstem conduction times in pups $[8,9]$. In addition, excess maternal intake of n-3 PUFAs was shown to cause abnormal ABRs in older adult offspring [10,18]. Further structural and functional analyses will be useful to reveal the mechanisms underlying the potential adverse effects of $n-3$ PUFAs.

\section{Limitations}

This study is not without limitations. First, only male mice were used in this study, so it is not known how the present findings would apply to females. Second, ABR thresholds of both WT and Fat-1 mice at 10 months were increased due to unknown reasons. Considering the fact that we performed the ABR measurements on 10-month-old mice more than 2 years after the initial ABR experiments $(2,13,16-17$ months old), we believe that the higher ABR thresholds at 10 months were likely caused by variability in epigenetic backgrounds related to the progression of AHL within the same C57BL/6 strain. Third, this study lacks the data of metabolome analysis because of technical problems. In this study, WT and Fat-1 mice were fed a standard rodent diet (CE2, Additional file 1: Table S2). As a result, the cochlear $n-6 / n-3$ ratio was predicted to be higher in WT mice than in Fat-1 mice. Since the n-6/n-3 ratio of Fat-1 mice is close to 1:1 in all organs/ tissues examined [11], we believe that the ratio is also close to 1:1 in Fat-1 mouse cochlea. Future studies should establish metabolomic data using cochlear tissue. Finally, a limitation of our histological analysis was the smaller animal numbers and larger variations in histological analyses that occurred compared with ABR analyses. Regarding the lack of correlation between the $A B R$ and histological results, we suspect that the $n-3$ PUFAs help to protect a wide range of the cochlea, not just on a specific cochlear region. However, it is also possible that additional histological assessments might reveal other reasons for the otoprotective effect of the n-3 PUFAs. Unfortunately, we are unable to conduct further animal experiments currently. The mechanism of the otoprotective effects of the n-3 PUFAs should be investigated further in future studies.

\section{Supplementary information}

Supplementary information accompanies this paper at https://doi. org/10.1186/s13104-019-4809-8.

Additional file 1: Fig. S1. Cdh23 genotyping and sequencing of Fat-1 mice. The Cdh 23 gene in three Fat- 1 transgenic mice was sequenced. All of the Fat- 1 transgenic mice examined had the same $C d h 23^{753 \mathrm{~A} / 753 \mathrm{~A}}$ genotype. Table S1. Summary table for the two-way ANOVA. Table S2. Fatty acid composition of CE-2 diet. Additional methods. Cdh23 genotyping.

\section{Abbreviations}

ABR: auditory brainstem response; AHL: age-related hearing loss; ANOVA: analyses of variance; DHA: docosahexaenoic acid; n.s.: no significant difference; OHC: outer hair cell; PUFA: polyunsaturated fatty acid; SG: spiral ganglion; SGN: spiral ganglion neuron; SPL: sound pressure level; SV: stria vascularis; WT: wild-type.

\section{Acknowledgements}

We thank Dr. J.X. Kang, Dr. M. Arita, and Dr. T. Naganuma for providing us with the Fat-1 transgenic mice, Dr. S. Someya for technical advice regarding Cdh23 genotyping and sequencing, Ms. S. Mochizuki, Ms. N. Shibata, and Ms. F. Suzuki for contributing to the histological analyses, and Ms. E. Kiryu and Ms. S. Makino for animal care.

\section{Authors' contributions}

JS and NO designed the research, $\mathrm{YH}$ and JS performed the research, JS and $\mathrm{NO}$ analyzed the data, NS, TK, YK, HI, and NO contributed reagents/materials/ analysis tools, and $\mathrm{YH}, \mathrm{JS}, \mathrm{NS}$, and NO wrote the paper. All authors have read and approved the manuscript.

\section{Funding}

This work was supported by Grant-in-Aid for Scientific Research (C) from Ministry of Education, Culture, Sports, Science and Technology Japan (K16K11700, J.S.), Grant-in-Aid for Scientific Research (B) from Ministry of Education, Culture, Sports, Science and Technology Japan (\#21300115, N.O.), Grant-in-Aid for Scientific Research on Innovative Areas from Ministry of Education, Culture, Sports, Science and Technology Japan (17H06059, N.S.), Research Grant from the Asahi Glass Foundation (N.O.), Research Fellowship of the Japan Society for the Promotion of Science for Young Scientists from the Japan Science and Technology Agency (N.S.), and Scientific Research Promotion Grant from the Mishima Kaiun Memorial Foundation (N.S.).

\section{Availability of data and materials}

Datasets used and analyzed for the current study are available from the corresponding author upon reasonable request.

\section{Ethics approval and consent to participate}

All experimental procedures were approved by Tohoku University's committee for animal experimentation (MED\#2013-114) and conducted in accordance with the National Institutes of Health Guide for the Care and Use of Laboratory Animals.

Consent for publication

Not applicable.

Competing interests

The authors declare that they have no competing interests. 


\section{Author details}

${ }^{1}$ Department of Otolaryngology-Head and Neck Surgery, Tohoku University School of Medicine, 1-1 Seiryo-machi, Aoba-ku, Sendai, Miyagi 980-8574, Japan. ${ }^{2}$ Department of Molecular Genetics, Institute of Biomedical Sciences, Fukushima Medical University, 1 Hikarigaoka, Fukushima 960-1295, Japan. ${ }^{3}$ Department of Developmental Neuroscience, Centers for Neuroscience, Tohoku University School of Medicine, 2-1 Seiryo-machi, Aoba-ku, Sendai, Miyagi 980-8574, Japan. ${ }^{4}$ Laboratory of Rehabilitative Auditory Science, Tohoku University Graduate School of Biomedical Engineering, 1-1 Seiryou-machi, Aoba-ku, Sendai, Miyagi 980-8574, Japan.

Received: 22 August 2019 Accepted: 14 November 2019

Published online: 26 November 2019

\section{References}

1. Yamasoba T, Lin FR, Someya S, Kashio A, Sakamoto T, Kondo K. Current concepts in age-related hearing loss: epidemiology and mechanistic pathways. Hear Res. 2013;303:30-8.

2. Gates GA, Mills JH. Presbycusis. Lancet. 2005;366:1111-20.

3. Yurko-Mauro K, McCarthy D, Rom D, Nelson EB, Ryan AS, Blackwell A, et al. Beneficial effects of docosahexaenoic acid on cognition in age-related cognitive decline. Alzheimers Dement. 2010;6:456-64.

4. Bazinet RP, Laye S. Polyunsaturated fatty acids and their metabolites in brain function and disease. Nat Rev Neurosci. 2014;15:771-85.

5. Gopinath B, Flood VM, Rochtchina E, McMahon CM, Mitchell P. Consumption of omega-3 fatty acids and fish and risk of age-related hearing loss. Am J Clin Nutr. 2010;92:416-21.

6. Dullemeijer C, Verhoef P, Brouwer IA, Kok FJ, Brummer RJ, Durga J. Plasma very long-chain $n-3$ polyunsaturated fatty acids and age-related hearing loss in older adults. J Nutr Health Aging. 2010;14:347-51.

7. Curhan SG, Eavey RD, Wang M, Rimm EB, Curhan GC. Fish and fatty acid consumption and the risk of hearing loss in women. Am J Clin Nutr. 2014:100:1371-7.

8. Haubner LY, Stockard JE, Saste MD, Benford VJ, Phelps CP, Chen LT, et al. Maternal dietary docosahexanoic acid content affects the rat pup auditory system. Brain Res Bull. 2002;58:1-5.

9. Stockard JE, Saste MD, Benford VJ, Barness L, Auestad N, Carver JD. Effect of docosahexaenoic acid content of maternal diet on auditory brainstem conduction times in rat pups. Dev Neurosci. 2000;22:494-9.
10. Church MW, Jen KL, Anumba Jl, Jackson DA, Adams BR, Hotra JW. Excess omega-3 fatty acid consumption by mothers during pregnancy and lactation caused shorter life span and abnormal ABRs in old adult offspring. Neurotoxicol Teratol. 2010;32:171-81.

11. Kang JX, Wang J, Wu L, Kang ZB. Transgenic mice: fat-1 mice convert n-6 to n-3 fatty acids. Nature. 2004;27:504.

12. Sakayori N, Kikkawa T, Tokuda H, Kiryu E, Yoshizaki K, Kawashima H, et al. Maternal dietary imbalance between omega- 6 and omega-3 polyunsaturated fatty acids impairs neocortical development via epoxy metabolites. Stem Cells. 2016;34:470-8.

13. Suzuki J, Oshima T, Yoshida N, Kimura R, Takata Y, Owada Y, et al. Preservation of cochlear function in Fabp3 ( $\mathrm{H}-\mathrm{Fabp})$ knockout mice. Neurosci Res. 2014;81-82:64-8.

14. Johnson KR, Tian C, Gagnon LH, Jiang H, Ding D, Salvi R. Effects of Cdh23 single nucleotide substitutions on age-related hearing loss in C57BL/6 and 129S1/Sv mice and comparisons with congenic strains. Sci Rep. 2017;7:44450.

15. Han C, Linser P, Park HJ, Kim MJ, White K, Vann JM, et al. Sirt1 deficiency protects cochlear cells and delays the early onset of age-related hearing loss in C57BL/6 mice. Neurobiol Aging. 2016;43:58-71.

16. Yang $\mathrm{CH}$, Schrepfer T, Schacht J. Age-related hearing impairment and the triad of acquired hearing loss. Front Cell Neurosci. 2015;9:276.

17. Martinez-Vega R, Partearroyo T, Vallecillo N, Varela-Moreiras G, Pajares MA, Varela-Nieto I. Long-term omega-3 fatty acid supplementation prevents expression changes in cochlear homocysteine metabolism and ameliorates progressive hearing loss in $(57 \mathrm{BL} / 6 \mathrm{~J}$ mice. J Nutr Biochem. 2015;26:1424-33.

18. Church MW, Jen KL, Jackson DA, Adams BR, Hotra JW. Abnormal neurological responses in young adult offspring caused by excess omega-3 fatty acid (fish oil) consumption by the mother during pregnancy and lactation. Neurotoxicol Teratol. 2009;31:26-33.

\section{Publisher's Note}

Springer Nature remains neutral with regard to jurisdictional claims in published maps and institutional affiliations.

Ready to submit your research? Choose BMC and benefit from:

- fast, convenient online submission

- thorough peer review by experienced researchers in your field

- rapid publication on acceptance

- support for research data, including large and complex data types

- gold Open Access which fosters wider collaboration and increased citations

- maximum visibility for your research: over $100 \mathrm{M}$ website views per year

At BMC, research is always in progress.

Learn more biomedcentral.com/submissions 